\title{
Imposing Integrability in Geometric Shape-from-Shading
}

\author{
Mario Castelán^ and Edwin R. Hancock \\ Dept. of Computer Science, University of York, York YO10 5DD, United Kingdom \\ \{mario, erh\}@cs.york.ac.uk
}

\begin{abstract}
This paper describes a Fourier domain algorithm for surface height recovery using shape from shading. The algorithm constrains surface normals to fall on an irradiance cone. The axis of the cone points in the light source direction. The opening angle of the cone varies with iteration number, and is such that the surface normal minimizes brightness error and satisfies the integrability constraint. The results show that the method recovers needle maps that are both smooth and integrable, with improved surface stability.
\end{abstract}

\section{Introduction}

Shape-from-shading (SFS) is a problem in computer vision which has been an active topic of research for some three decades. The process was identified by Marr[10] as a key process in the computation of the 2.5D sketch, and was studied in depth by Horn[5]. The topic has also been the focus of recent research in the psychophysics literature [9] [2] [3]. Stated more formally, the SFS problem can be regarded as that of calculating the set of partial derivatives $\left(Z_{x}, Z_{y}\right)$ corresponding to a surface $Z=Z(x, y)$, where $Z$ is simply an intensity image 11 In brief, we need to solve the image irradiance equation, $E(x, y)=R(p(x, y), q(x, y), s)$, where $E$ is the intensity value of the pixel with position $(x, y), R$ is a function referred to as the reflectance map [6], that maps the surface gradients $p=\frac{\partial Z(x, y)}{\partial x}$ and $q=\frac{\partial Z(x, y)}{\partial y}$ to an intensity value and $s$ is the light source direction. If the surface normal at the location $(x, y)$ is $n=(p, q,-1)$ then under Lambertian reflectance model, the image irradiance equation becomes $E(x, y)=n \cdot s$.

Unfortunately, the image irradiance equation is underconstrained, and the family of surface normals fall on a reflectance cone whose apex angle $\alpha$ is equal to $\cos ^{-1} E(x, y)$, and whose axis points in the light source direction $s$. Several constraints have been used to overcome the underconstrained nature of the Lambertian shape-from-shading problem. However, their main drawback is that they have a tendency to oversmooth the recovered surface slopes and result in poor

\footnotetext{
* Supported by National Council of Science and Technology (CONACYT), Mexico, under grant No. 141485.

${ }^{1}$ More than one image can be used, but this is an extension of SFS referred to as photometric stereo.
} 
data-closeness. The net result is a loss of fine surface detail. For a complete survey of most SFS methods, see [16].

In a recent paper Worthington and Hancock 14 have demonstrated how these problems may be overcome by constraining the surface normals to lie on the reflectance cone and allowing them to rotate about the light source direction subject to curvature consistency constraints. Unfortunately, the needle maps delivered by the method are not guaranteed to satisfy the integrability constraint, which means that the recovered partial derivatives are not independent on the path of integration (i.e. the height function may not be recoverable). Besides, these needle maps also suffer the drawback of high dependency on the image intensities, making the method prone to noisy data such as specularities, roughness and overshadowed areas.

There are a number of ways in which a surface may be recovered from a field of surface normals 78 . One approach is to use trigonometry to increment the height function along a path or a front [112]. However, one of the most elegant approaches is that described by Frankot and Chellappa [4] which shows how the surface may be reconstructed subject to integrability constraints by performing a Fourier analysis of the field of surface normals.

The aim in this paper is to develop a shape-from-shading scheme that can be used to recover integrable needle maps subject to hard constraints on Lambertian reflectance as well as relaxing the image intensity dependance driven by such constraints. In order to demonstrate how the two techniques can be combined, in subsequent sections we will briefly explain the geometric approach developed by Worthington and Hancock 14 for solving SFS, as well as the algorithm proposed by Frankot and Chellapa [4] for enforcing integrability in SFS.

\section{Geometric Approach for SFS}

Worthington and Hancock [14] have developed an SFS method in which the image irradiance equation is treated as a hard constraint by constraining the recovered surface normals to lie on the reflectance cone. Suppose that $\hat{N}_{k}$ is a smoothed 2 surface normals at step $k$ of the algorithm, then the update equation for the surface normal directions is

$$
\hat{\hat{N}}_{k+1}=\Theta \hat{N}_{k}
$$

where $\Theta$ is a rotation matrix computed from the apex angle $\alpha$ and the angle between the current smooothed estimate of the surface normal direction $\tilde{N}_{k}$ and the light source direction. To restore the surface normal to the irradiance cone, it must be rotated by an angle

$$
\theta=\cos ^{-1}(E)-\cos ^{-1}\left(\frac{\hat{N}_{k} \cdot s}{\left\|\hat{N}_{k}\right\| \cdot\|s\|}\right)
$$

${ }^{2}$ For further details about the suggested method for smoothing the normal field, see 13 . 
about the axis $(u, v, w)^{T}=\tilde{N}_{k} \times s$. Hence, the rotation matrix is

$$
\Theta=\left(\begin{array}{ccc}
c+u^{2} c^{\prime} & -w s+u v c^{\prime} & v s+u w c^{\prime} \\
w s+u v c^{\prime} & c+v^{2} c^{\prime} & -u s+v w c^{\prime} \\
-v s+u w c^{\prime} & u s+v w c^{\prime} & c+w^{2} c^{\prime}
\end{array}\right)
$$

where $c=\cos (\theta), c^{\prime}=1-c$ and $s=\sin (\theta)$.

The needle maps delivered by this geometric framework have proved to be useful in experiments for topography-based object recognition [15].

\section{Integrability in SFS}

The integrability condition in SFS ensures that the recovered surface satisfies the following condition on the partial derivatives of the height function: $Z_{x y}=$ $Z_{y x}$. This condition can also be regarded as a smoothness constraint, since the partial derivatives of the surface need to be continuous in order that they can be integrable or independent on the path of integration. In 4] Frankot and Chellapa proposed a method to project a gradient field to the nearest integrable solution. They suggested to use a set of integrable basis functions to represent the surface slopes so as to minimize the distance between an ideally integrable gradient field and a non integrable one.

Following [4], if the surface $Z$ is given by

$$
\tilde{Z}(x, y)=\sum_{\omega \in \Omega} \tilde{C}(\omega) \phi(x, y, \omega)
$$

where $\omega$ is a two dimensional index belonging to a domain $\Omega$, and $\phi(x, y, \omega)$ is a set of basis functions which are not necessarily mutually orthogonal, the partial derivatives of $\tilde{Z}$ can also be expressed in terms of this set of basis functions using the formulae

$$
\tilde{Z}_{x}(x, y)=\sum_{\omega \in \Omega} \tilde{C}(\omega) \phi_{x}(x, y, \omega) \quad \text { and } \quad \tilde{Z}_{y}(x, y)=\sum_{\omega \in \Omega} \tilde{C}(\omega) \phi_{y}(x, y, \omega)
$$

Given that $\phi_{x}(x, y, \omega)$ and $\phi_{y}(x, y, \omega)$ are integrable, then so are the mixed partial derivatives of $\widetilde{Z}(x, y)$.

In the same way, the possibly non integrable gradient field (which, indeed, is the only information we have) can be represented as

$$
\hat{Z}_{x}(x, y)=\sum_{\omega \in \Omega} \hat{C}_{1}(\omega) \phi_{x}(x, y, \omega) \quad \text { and } \quad \hat{Z}_{y}(x, y)=\sum_{\omega \in \Omega} \hat{C}_{2}(\omega) \phi_{y}(x, y, \omega)
$$

Note that, as $\hat{C}_{1} \neq \hat{C}_{2}$, then $\hat{Z}_{x y} \neq \hat{Z}_{y x}$.

The goal then is to find the set of coefficients that minimize the quantity

$$
d\left\{\left(\hat{Z}_{x}, \hat{Z}_{y}\right),\left(\tilde{Z}_{x}, \tilde{Z}_{y}\right)\right\}=\iint\left\|\tilde{Z}_{x}-\hat{Z}_{x}\right\|^{2}+\left\|\tilde{Z}_{y}-\hat{Z}_{y}\right\|^{2} d x d y
$$


As Frankot and Chellapa proved, the set of coefficients $\tilde{C}(\omega)$ minimizing the distance given by the above equation is

$$
\tilde{C}(\omega)=\frac{P_{x}(\omega) \hat{C}_{1}(\omega)+P_{y}(\omega) \hat{C}_{2}(\omega)}{P_{x}(\omega)+P_{y}(\omega)},
$$

where $P_{x}(\omega)$ and $P_{y}(\omega)$ are $\iint\left\|\phi_{x}(x, y, \omega)\right\|^{2} d x d y$ and $\iint\left\|\phi_{y}(x, y, \omega)\right\|^{2} d x d y$ respectively.

If $\phi(x, y, \omega)$ is assumed to be the set of Fourier basis functions $\exp \left(j \omega_{x} x+\right.$ $\left.j \omega_{y} y\right)$, with $\Omega=(2 \pi n, 2 \pi m)$, where $n \in\{0,1, \cdots, N-1\}$ and $m \in\{0,1, \cdots, M-$ 1) for an $N \times M$ image, then $P_{x}=\omega_{x}^{2}, P_{y}=\omega_{y}^{2}, \hat{C}_{1}(\omega)=\hat{C}_{x}(\omega) / j \omega_{x}$, and $\hat{C}_{2}(\omega)=\hat{C}_{y}(\omega) / j \omega_{y}$. Therefore, (8) is represented in the Fourier domain by

$$
\tilde{C}(\omega)=\frac{-j \omega_{x} \hat{C}_{x}(\omega)-j \omega_{y} \hat{C}_{y}(\omega)}{\omega_{x}^{2}+\omega_{y}^{2}} .
$$

In this manner, by projecting the set of coefficients $\tilde{C}(\omega)$ back to the spatial domain, a height map corresponding to the nearest integrable surface $\tilde{Z}(x, y)$ can be obtained from the input gradient field.

\section{Introducing the Integrability Condition in the Geometric Approach for SFS}

The idea underpinning this paper is to calculate the nearest integrable surface and obtain the apex angle of the Lambertian cone on this surface after each iteration. The algorithm can be summarized as follows:

1. Calculate an initial estimate of surface normals $\mathrm{N}=\left(N_{x}, N_{y}, N_{z}\right)$.

2. Smooth $N$ to obtain $\hat{N}$.

3. Obtain the nearest integrable surface $\tilde{Z}$ by solving (9) using the smoothed surface normal field $\hat{N}$.

4. Get the apex angle $\alpha$ of the Lambertian cone using the values of $\tilde{Z}$, that is to say, $\alpha=\cos ^{-1}(\tilde{Z})$.

5. Calculate $\hat{\hat{N}}$, by rotating $\hat{N}$, using (11).

6. Make $N=\hat{\hat{N}}$ and return to step 2. Repeat until a desired number of iterations has been reached.

We should note that in this method the rotation matrix does not remain static through the iterative process, since the changes in $\alpha$ depend on the recovered surface after each iteration. It is also important to remark that due to the projection of the surface normals to the reflectance cone after each iteration, the z-component $\hat{\hat{N}} z$ of the normal $\hat{\hat{N}}$ will always correspond to the calculated height surface of the final gradient field when using the Frankot and Chellapa height recovery method. By contrast, in the method of Worthington and Hancock the z-component $N z$ will always be the normalised input intensity image. Therefore, besides calculating surface gradients, the new algorithm also calculates height information. 


\section{Experiments}

The algorithm was tested on synthetic as well as real images. The evaluation criteria was based on the absolute height difference and degree of gradient consistency (i.e. the percentage of pixels of every image whose differences $Z_{x y}-Z_{y x}$ are less than or equal to a certain threshold 3 ). In our experiments we have compared the results obtained with the geometric approach of Worthington and Hancock, and the new integrable-geometric approach.

Three synthetic images were tested]. Forty real world images (fifteen of these with corresponding height data, taken from the range database in [17], and the rest taken from [11] and from [16]) were also used for test 5 .
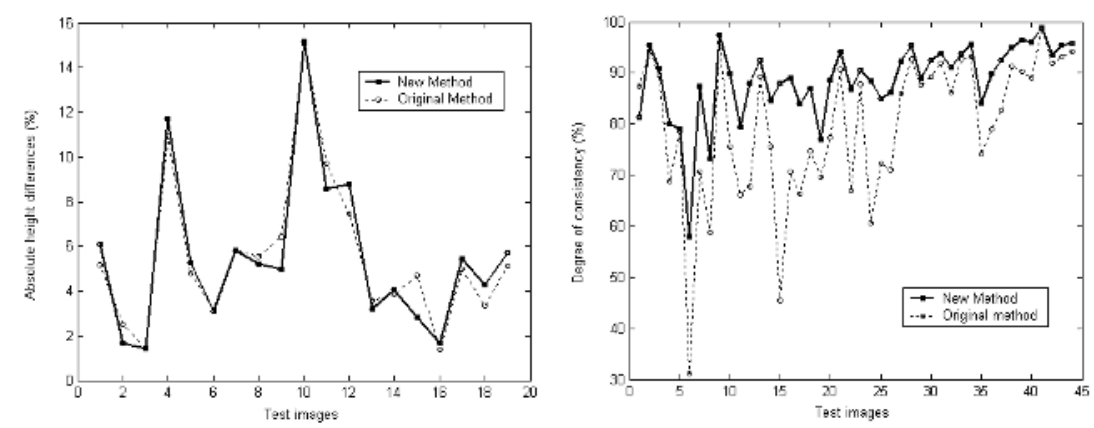

Fig. 1. Left: plot of the absolute height differences for synthetic and range images. Right: plot of the gradient consistency degree tests.

Figure1(left) shows the results for the absolute height differences. The original approach is represented by the dotted line, while the new one is represented by the solid line. The plot reveals that there seems to be no bias for favoring each method, and that the height difference between them is not significant.

The results of the experiments for degree of gradient consistency are summarized in Figure 1 (right). The figure shows that the combined algorithm (solid line) gives more consistent results than the original one (dotted lined), as the percentage of gradient consistency is greater for the new approach. This suggests that the new method is enforcing integrability in the original method.

In a further analysis of the results, Figure 2 shows a 3D plot of the recovered heights for each method. The first column corresponds to the input image, the second column is a plot of the range data of each image given as a base for comparison, the third column represents the recovered height map for the new method and the fourth column shows the height maps for the original method.

\footnotetext{
${ }^{3}$ For all the experiments this threshold was set to 0.1 .

${ }^{4}$ These images were also used in [16].

${ }^{5}$ For all the tests, the light source direction was assumed to be $[0,0,1]$.
} 

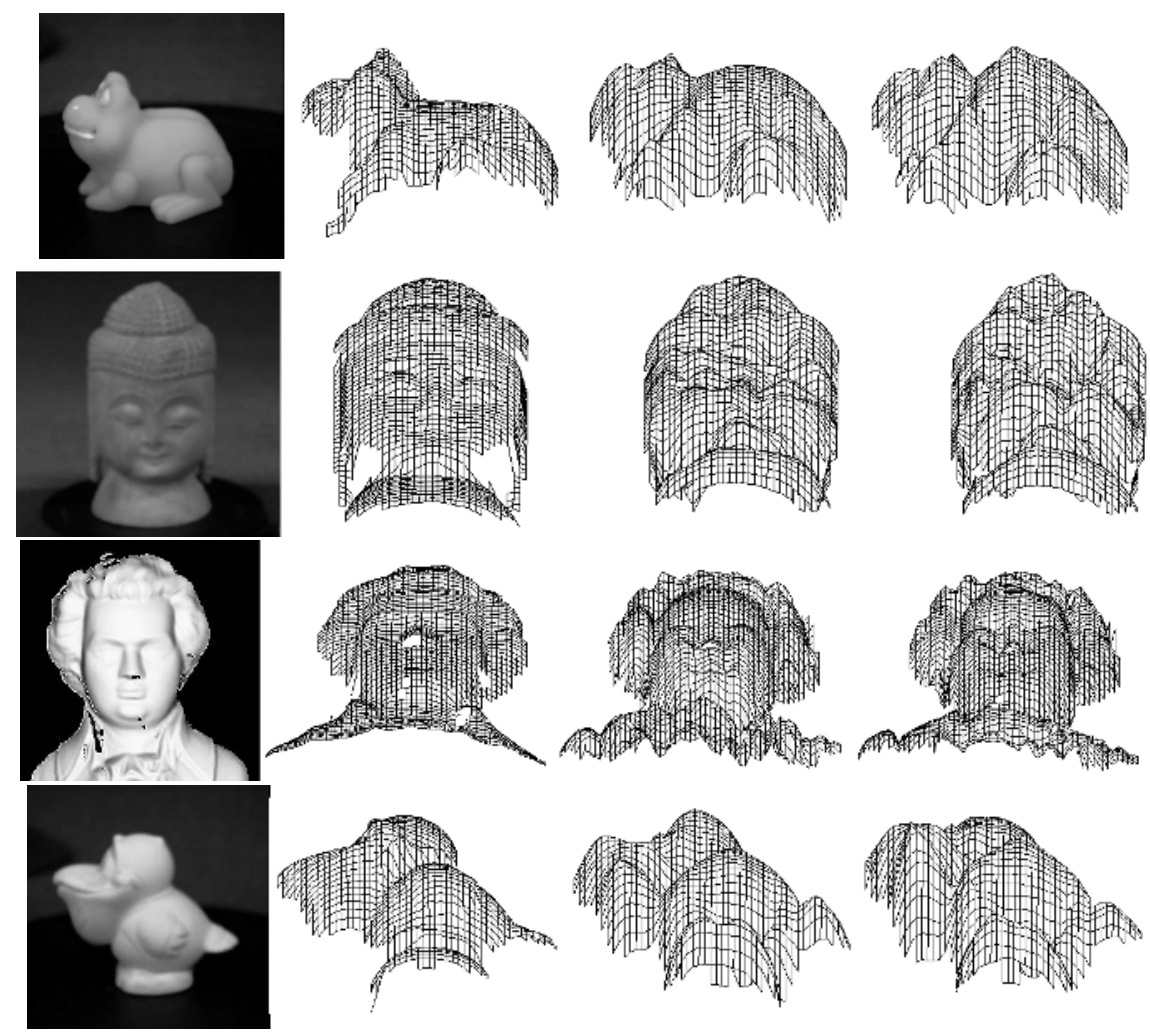

Fig. 2. Recovered height surface for range images. From left to right: intensity image, range data, recovered surface for the combined algorithm, recovered surface for the original algorithm.

We can observe that the new algorithm seems to stabilize the surface, avoiding some of the sudden changes present in the recovered surface for the original method. Specifically, in the cases of the frog and the pelican, the recovered surface appears to be smoother, with none of the spurious peaks in the height map which result from the use of the original method. Also, the height plots of Budda and Mozart show a more stable surface than those produced by the original method.

Figure 3 shows the recovered needle maps for each method. A visual examination of the results suggests that the new method delivers needle maps that are both smoother and contain more fine topographic detail than original method. This effect is more evident in the cases of the frog and Budda. 

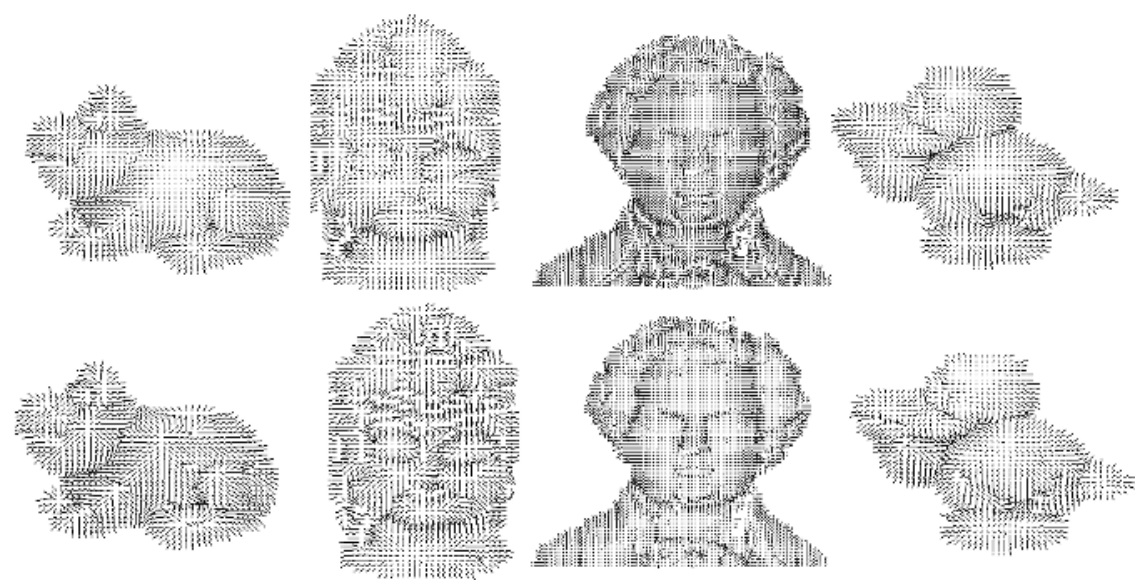

Fig. 3. Recovered needle maps for each method. Top row: combined algorithm. Bottom row:original algorithm.

\section{Conclusions}

In this paper we have demonstrated how to impose integrability constraints on the geometric approach for SFS suggested by Worthington and Hancock. We follow Frankot and Chellapa and impose the constraints in the Fourier domain. Experiments reveal that the resulting method exhibits improved robustness and gradient consistency. However, although the height difference statistics do not reveal any systematic improvement in algorithm performance, both the recovered height surfaces and the needle maps delivered by the new algorithm appear to be better behaved and also preserve fine surface detail. It is important to comment that in this new method the calculation of surface orientations is less constrained by the irradiances of the image, as the rotation matrix changes through the iterative process. This is a way of relaxing the original method's problem of hard constraints on data-closeness with the image irradiance equation. Our future plans include using alternative basis functions and in particular the discrete cosine transform, as well as comparing the output needle maps for local integration tests.

\section{References}

1. A.G. Bors, E.R. Hancock, R.C. Wilson. Terrain Analysis Using Radar Shapefrom-Shading. IEEE Trans, on Pattern Analysis and Machine Intelligence, 25(5), 2003.

2. R.G.F. Erens, A.M.L. Kappers and J.J. Koenderink. Perception of Local Shape from Shading. Perception and Psychophysics, 54(2):145-156, 1993.

3. R.G.F. Erens, A.M.L. Kappers and J.J. Koenderink. Estimating Local Shape from Shading in the Presence of Global Shading. Perception and Psychophysics, 54(3):334-342, 1993. 
4. R.T. Frankot and R. Chellapa. A Method for Enforcing Integrability in Shape from Shading Algorithms. IEEE Trans. Pattern Analysis and Machine Intelligence, 10(4):438-451, 1988.

5. B.K.P. Horn and M.J. Brooks Shape from Shading. MIT Press, Cambridge, MA, 1989.

6. B.K.P. Horn. Understanding Image Intensities. Artificial Intelligence, 8:201-231, 1977.

7. R. Klette and K. SChluns, Height Data from Gradient Fields. Proc. Machine Vision Applications, Architectures and Systems Integration. 5:204-215, 1996.

8. R. Klette, K. Schluns, and A. Koschan. Computer Vision: Three-dimensional Data From Images. Springer-Verlag Singapore, 1998.

9. J.J. Koenderink, A.J. Van Doorn and A.M.L. Kappers. Surface Perception in Pictures. Perception and Psychophysics, 52(5):487-496, 1992.

10. D. Marr. Vision. W.H. Freeman \& Co., San Francisco, 1982.

11. S.A. Nene, S.K. Nayar, and H. Murase. Columbia Object Image Library: COIL100. Technical Report CUCS-006-96, Department of Computer Science, Columbia University, February 1996.

12. A. Robles-Kelly and E.R. Hancock. A Graph-Spectral Method for Surface Height Recovery from Needle-maps. Proceedings of the IEEE Conference on Computer Vision and Pattern Recognition, IEEE Computer Society, 2001.

13. P.L. Worthington and E.R. Hancock. Needle Map Recovery Using Robust Regularizers. Image and Vision Computing, 17(8):545-559, 1998.

14. P.L. Worthington and E.R. Hancock. New Constraints on Data-closeness and Needle Map Consistency for Shape-from-shading. IEEE Trans. on Pattern Analysis and Machine Intelligence, 21(12):1250-1267, 1999.

15. P.L. Worthington and E.R, Hancock. Object Recognition Using Shape-fromShading. IEEE Trans. on Pattern Analysis and Machine Intelligence, 23(5):535542, 2001.

16. R. Zhang, P.S. Tsai, J.E. Cryer and M. Shah. Shape from Shading: A Survey. IEEE Trans. on Pattern Analysis and Machine Intelligence, 21(8):690-706, 1999.

17. Signal Analysis and Machine Perception Laboratory, Department of Electrical Engineering, The Ohio State University, http://sampl.eng.ohio-state.edu/sampl/data/3DDB/RID/index.htm 